Del Real Villarreal 50

DOI: https://doi.org/10.29105/gmjmx17.33-3

Artículos

\title{
MEDIACIONES TRANSVERSALES EN LA CONSTRUCCIÓN SOCIAL DE LAS MUJERES EJECUTIVAS
}

\section{TRANSVERSAL MEDIATIONS IN THE SOCIAL CONSTRUCTION OF FEMALE BUSINESS MANAGERS}

\author{
Lilia Paola Del Real Villarreal \\ Tecnológico de Monterrey, México \\ ORCID: https://orcid.org/0000-0001-5577-9291
}

Autor para correspondencia: Lilia Paola Del Real Villarreal, email: paoladelreal@gmail.com

\section{Resumen}

Texto justificado en investigación teórica acerca de las mediaciones que reciben las mujeres ejecutivas de alta gerencia a lo largo de su vida y su entretejido en el camino de la crianza, relación con instituciones y medios de comunicación, con el objetivo de exponer las mediaciones de la familia, organización y medios de comunicación, así como su repercusión en la construcción social de las mujeres. El método de selección de las investigaciones incluidas fue a través de la pertinencia con el tema y la representación de las mujeres que actualmente ocupan los puestos ejecutivos. La mediación de la familia y la organización son fundamentales en la construcción social de la mujer de alta gerencia. Los medios de comunicación ocupan una relevancia distinta en el proceso, afectando en la creación de estereotipos disonantes entre la femineidad y el liderazgo.

Palabras clave: mediaciones, familia, organización, construcción social de las mujeres, medios de comunicación.

\begin{abstract}
Theorical investigation about the mediations that female business managers receive throughout their lives and how these are intertwined with parenting, institutional relationships, and mass media. The investigation objective is to expose family, organization, and mass media mediations, as well as its repercussions in senior management women social construction. The method of selection of the research included was through relevance to the topic and representation of women currently in managerial positions. Family and organizational mediations are fundamental in the social
\end{abstract}

Global Media Journal México 17(33). julio - diciembre 2020. Pp. 50-71. 
construction of women that occupy senior management roles. The mass media mediations bring a different relevance, in the creation of dissonant stereotypes between femininity and leadership.

Keywords: mediations, family, organization, women social construction, media.

Recibido: 06/10/2020

Aceptado: 11/12/2020

\section{Introducción}

Esta investigación profundiza en las diferentes mediaciones que se entretejen transversalmente en la vida de las mujeres de alta gerencia. ${ }^{1}$ El 40 por ciento de las mujeres que dirigen organizaciones en México tienen entre 25 y 44 años (Inegi, 2020), edad que se traslapa con la fase de resistencia (entre los 36 y 45 años) de acuerdo con las etapas del desarrollo profesional de las mujeres investigadas por Velázquez (2012) y O’Neil y Bilimoria (2005), en donde se explora su vida laboral y personal. En estos estudios se argumenta que durante este periodo se toman decisiones entre los roles que deben cumplir como madres y la satisfacción que encuentran en su trabajo.

Las mediaciones que han afectado a estas mujeres se han experimentado desde la infancia y hasta su adultez, siendo las mediaciones de los primeros años las más

\footnotetext{
${ }^{1}$ Cantidad pequeña de personas en la organización que son responsables de establecer políticas de operaciones, dirigen la interacción y organización
}

importantes en la construcción de su personalidad (Bertaux-Waine, 1993). Ellas crearon imaginarios por distintos conductos, como la familia, la organización y los medios masivos de comunicación, de los cuales se profundiza en los siguientes apartados de acuerdo con su influencia en las mujeres de alta gerencia y se incorporan estudios relevantes, encontrando que unas mediaciones fueron más significativas que otras.

La organización está presente en el hogar por medio de los discursos de los padres durante la infancia, socialización del lenguaje y de las expectativas familiares, que posteriormente intervienen de manera directa en la toma de decisiones y comportamientos laborales en la vida adulta (Paugh, 2005; Lucas, 2011; Lair, 2007). Los medios masivos de comunicación también forman parte de la vida cotidiana y representan los estereotipos femeninos y masculinos para una gran población que replica conductas y de su equipo; pueden ser gerentes, subdirectoras o directoras (Villasmil, 2007). 
comportamientos (García, 2014; Cuadrado, 2007).

Las mujeres de alta gerencia se autorrepresentan mediante recursos de capital psicológico para la consecución de metas (Machín-Rincón et al., 2020), comunicación clara, directa y franca (Cárdenas de Santamaría et al., 2013) y la aceptación de retos de todo tipo, lo cual incluye el balance entre su familia y su trabajo (Bernal y Taracena, 2013), características que contrastan con los estereotipos femeninos de los medios masivos de comunicación, relacionados con la subordinación, sumisión y dependencia (Rocha-Sánchez y Díaz-Loving, 2005; Bonavitta y Garay Hernández, 2014; García, 2014) y se identifican más, con las características masculinas o instrumentales representadas en los varones (Gherardi, 1995; Parsons, 1955).

Los recursos y características de las mujeres de alta gerencia son adquiridos a través de las mediaciones. El presente artículo tiene el objetivo de exponer las mediaciones de la familia, organización y medios de comunicación, así como su repercusión en las ejecutivas. El método de selección de las investigaciones incluidas fue a través de la pertinencia con el tema y la representación de las ejecutivas que actualmente ocupan los puestos de poder en la organización, para así

\footnotetext{
${ }^{2}$ Los estudios de Martín-Barbero (1986) y Orozco
} (1991, 1997) están especializados en la televisión, encuadrar el conjunto de factores que apoyan o limitan su desarrollo profesional.

\section{Mediaciones transversales en las mujeres}

\section{Mediaciones de los medios masivos de comunicación}

Martín-Barbero $^{2} \quad$ (1986) define las mediaciones como el lugar donde se otorga el sentido a la comunicación, menciona que la comunicación se convierte en una cuestión de cultura más que de medios masivos de comunicación. Las mediaciones son las herramientas por las cuales los individuos construyen su identidad (Martín-Barbero, 1986; Orozco, 1997), y manejan su adaptación al estilo de vida impuesto por la cultura dominante.

Orozco (1991) y Beauvoir (1981) coinciden en sus postulados sobre construcción social del individuo. Orozco (1991) menciona que los niños como televidentes no nacen, sino que se hacen a través de la intervención implícita o explícita de diversos agentes socializadores, Beauvoir (1981) menciona que las mujeres no nacen, sino se hacen, a través de las normas socializadoras, de un estado hegemónico.

Los medios masivos de comunicación son formadores de ideología, en conjunto con su

en este estudio se utilizarán también para otros medios masivos de comunicación. 
labor de informar y entretener, son grandes constructores de personalidad, formando deseos, intereses y aspiraciones. El consumo televisivo nutre las identidades femeninas contemporáneas (Bonavitta y Garay Hernández, 2014; Padilla, 2005), específicamente en las mismas mujeres telespectadores que observan más de cuatro horas diarias un medio de comunicación (García, 2014).

De los Ríos (1997) responsabiliza a los medios masivos de comunicación de convertir a las niñas en figuras para agradar, lo que les impide desarrollar una correcta autoestima. "Esto está permitiendo que la imagen que se crea cada persona de sí misma sea construida a través de modelos existentes sexistas predeterminados, anulando las capacidades y potencialidades que tenemos las personas como seres humanos" (p. 99). Vega (2006) fortalece este postulado desde la perspectiva de género y observa que detrás de las diferencias sexuales entre ser hombre y ser mujer, se han elaborado desigualdades sociales, políticas, económicas y culturales, consecuencia de los flujos de información asimétrica en los discursos de roles de ambos sexos.

De acuerdo con los estudios de género en la recepción televisiva y el moldeamiento de conductas, para Bonavitta y Garay Hernández (2014)

$$
\begin{aligned}
& \text { El género constituye una } \\
& \text { representación total del sistema }
\end{aligned}
$$

social, en tanto lugar en el que se cruzan los discursos, creencias y normas sobre lo que la identidad femenina y masculina representa $y$ sobre la relación de poder que priva entre ambos géneros, y que se ha traducido históricamente en la supremacía masculina sobre la subordinación femenina (p. 19).

Rocha-Sánchez y Díaz Loving (2005) definen los estereotipos en México impuestos por los medios masivos de comunicación, en donde el hombre tiene un rol instrumental, que se traduce en actividades laborales de producción con el objetivo de mantener a la familia, con características primordiales de autonomía, orientación al logro, fuerza, éxito y ser proveedor. Por su parte, la mujer es representada con las características dirigidas al cuidado de los hijos, el hogar, la pareja, así como cualidades de sumisión, dependencia y abnegación.

Las audiencias mexicanas de los años ochenta y noventa contemplaron estos estereotipos de manera permanente, ya que existía un mono consumo televisivo por la situación socioeconómica del país, por lo tanto, estaban apartados de la cultura del teatro, cine, museos, exposiciones, y otras atracciones (Orozco, 1997). Sin embargo, hoy en día la programación ha cambiado, las opciones son infinitas y las mujeres tienen más opciones de contenidos de entretenimiento. El consumo se ha vuelto optativo en relación con 
el engagement de las personas con los contenidos.

Anderson (2006) describe la desintegración de las tradicionales audiencias masivas, lo cual implica una creciente importancia de los escenarios de cola larga. Estos escenarios consisten en el interés de un mercado o de una gran audiencia alrededor de una pequeña selección de opciones de productos o contenidos, llamados hits, así como una larga cola de pequeñas audiencias interesadas en la infinidad de opciones de contenido que responden a las preferencias personales de los consumidores.

Desde esta realidad los medios han adquirido una presencia permanente y una posición central frente a las audiencias, no solo mediante los medios tradicionales, sino a través de un sinnúmero de contenidos digitales, en donde cada uno de ellos presenta propuestas distintas de comportamiento, apropiación del género y construcción social de los individuos entretejidos en los contenidos, otorgando a cada audiencia, una mediación para identificarse y replicar conductas y comportamientos afines a su realidad.

A través del modelo de mediaciones en la recepción televisiva, Orozco (1991) aporta el enfoque de identificar y discutir a fondo las mediaciones cognoscitivas, culturales, situacionales, estructurales y videotecnológicas, que complejizan la experiencia televisiva y describe las multimediaciones: 1) mediaciones de recepción televisiva, 2) la mediación cultural, 3) la mediación situacional o de referencia, 4) mediaciones institucionales, y 5) mediaciones videotecnológicas.

Wagner y Wodak (2006) en su análisis de las portadas de Cosmopolitan, definen que el éxito femenino está basado en la juventud, la belleza y el ser eficiente y femenina al mismo tiempo, una imagen segura de sí misma, pero sin ser amenazante; por otro lado, en Koller (2004) en su estudio de la revista Bussiness Week revela que la información presentada es como si los dos sexos estuvieran en competencia permanente, exponiendo el trabajo de la mujer en una metáfora del juego de la guerra entre los hombres y las mujeres, con una visión hegemónica de ejecutivos como soldados, independientemente del sexo.

Desde el enfoque de las mediaciones multiculturales, los medios de comunicación se entretejen en la recepción de las audiencias y esto genera imaginarios en las mujeres. En la presente investigación, los medios masivos de comunicación no se caracterizan como la mayor influencia en las mujeres ejecutivas, ya que las características femeninas que proponen los medios masivos de comunicación, contrastan con las que autorrepresentan las mujeres ejecutivas (Cuadrado, 2004; García, 2014), esto coincide con Del Real y Maeda (2020) quienes mencionan en su investigación sobre mujeres ejecutivas, que los medios masivos de 
comunicación tradicionales no tuvieron la misma relevancia que las mediaciones familiares y organizacionales en la formación de las mujeres ejecutivas, sin embargo, sí forman parte de su contexto general.

\section{Mediación familiar}

La dinámica familiar de infancia, así como la familia vigente, tiene intervenciones directas en el comportamiento de las mujeres. MartínBarbero (2003) define tres lugares de mediación: la cotidianidad familiar, la temporalidad social y la competencia cultural. Estos lugares moldean la forma de recibir la mediación y delimitan los constructos de realidad que se apropian.

La familia es el primer núcleo en donde se desarrolla el individuo y, por ende, tiene una injerencia fundamental en el modelamiento de la personalidad de cada ser humano que la compone. Coller (1991) entiende a la familia "más que como un agregado de individuos, como un conjunto de relaciones entre seres humanos unidos por algún lazo de parentesco o afectividad y que realizan alguna función en común” (p. 94). Demarchi et al. (2015) la describen como un escenario de interacción, "en el que se transmiten valores, normas y pautas de comportamiento que van configurando el comportamiento del individuo. Puede ser conformada por vínculos sanguíneos, jurídicos (matrimonio/adopción) o por alianzas o pactos que se tejen entre sus integrantes" (p. 126). Existen diferentes tipos de familias y estas como mediación imprimen en los niños, futuros adultos, conductas y comportamientos que responden a la dinámica del hogar.

La familia es fundamental en el estudio de la interacción social porque "representa a la vez un modelo y un ejemplo del sistema interactivo que implica una especialización de roles y actitudes, una relación de lugar, normas relacionales y un conjunto de representaciones y valores que orientan las conductas" (Dominique, 1992, p. 155). Esta genera un contexto con información, valores, creencias y estilos propios de vivir; construye una perspectiva desde la cual observar al mundo, a través de su vocabulario, acceso a insumos culturales y cosmovisión; es un núcleo de socialización reconocido de gran importancia, porque cotidianamente se van proporcionando las bases en el desarrollo infantil, influyendo en la construcción de la identidad (Herrera y Romero, 2014).

Múltiples investigaciones (Simpson, 1962; Serravallo, 2004; Lucas, 2011) describen el papel central de la comunicación de los padres en las aspiraciones de los hijos y su desempeño académico y laboral, comprobando que los jóvenes a quienes se les habían marcado valores de crecimiento económico, expectativas de terminar el bachillerato y una carrera profesional, tienen una movilidad 
social ascendente con más relevancia en su futuro.

Ochs (1988) describe el paradigma de socialización del lenguaje en los niños y la forma en que ellos construyen su lenguaje. El tipo de discurso y narrativa que se comparte en casa es el formato de pensamiento con el que ellos perciben la vida, socializan y generan comportamientos. Se ha investigado la socialización del lenguaje y se ha comprobado que los niños ganan competencias lingüísticas $\mathrm{y}$ conciben funciones laborales, a través del discurso permanente en la comunicación del hogar, como observadores y participantes cuando escuchan a sus cuidadores narrando sus rutinas laborales (Paugh, 2005). En este discurso, las mujeres construyen motivaciones y metas para el futuro, en donde se va delineando la elección de vida y carrera (Bertaux y Thompson, 1993).

Lair (2007) menciona que los individuos son socializados en una ideología de trabajo, del rol de las organizaciones en su contexto y esto eventualmente se convierte en parte de sus elecciones de vida y carrera profesional. Los diálogos que se dan en el momento de la cena (Paugh, 2005), las historias que se narran (Langellier y Peterson, 2006), pueden enseñarles a los niños qué tipo de trabajos son valorados, y de esta manera se construyen imaginarios sobre qué tipo de resultados se esperan de ellos en su futuro, estableciendo parámetros para guiar sus aspiraciones y expectativas. Lo anterior se complementa con el discurso de Lair (2007), quien propone que la socialización organizacional, es una cuestión de toda la vida y se desarrolla mucho antes de encontrar la relación laboral.

La comunicación familiar es, de acuerdo con Paugh (2005) un factor crítico para la comprensión de las ideologías sobre el trabajo, el manejo de las expectativas, horarios, cantidad de horas entregadas al oficio, obligaciones y conflictos para reconciliar la familia y los horarios laborales. La comunicación sobre el trabajo y las expectativas laborales en la familia se da en los siguientes escenarios (Paugh, 2005):

1. En la preparación para ir a trabajar, cuando los padres les explican a sus hijos, el por qué van a la oficina y cuál es la razón por la que usan ropa específica, las consecuencias de llegar tarde y las implicaciones generales que significa ir a un espacio laboral.

2. En el regreso a casa, cuando los padres continúan hablando sobre las actividades del trabajo.

3. En el momento de tener los alimentos en familia, en donde se comparten historias, se ponen al corriente de su día, la forma en que solucionaron sus problemas y su jornada laboral en general, como plática cotidiana.

4. En el momento en que el trabajo remunerado entra a la vida familiar, 
cuando los padres tienen que trabajar desde casa, hacer cuestiones de papeles, llamadas, correos electrónicos o visitas a clientes, todo esto contextualiza a los niños en los eventos relacionados con la vida laboral.

5. En los momentos de deportes, labores domésticas o la realización de la tarea académica, cuando los padres dan ejemplos o facilitan los procesos.

Este sinnúmero de momentos de socialización del lenguaje y familia genera las mediaciones familiares, a través de las cuales los niños se apropian de los mensajes y las conductas que los padres o cuidadores transmiten, contribuyendo en la conformación de su personalidad.

Los estudios también profundizan en el camino de los mensajes, la manera en que se emiten y quiénes los emiten. Bradford et al. (2001), encuentran que las mujeres de su investigación recibieron mensajes directos que sus madres y sus padres les comunicaron mediante el ejemplo. Otro elemento importante en la configuración de la personalidad es lo que se afirma del rol de género, ligado a la clase social.

Los sectores medios han sido más permeables a nuevas formas de organización y convivencia familiar, distanciándose de los modelos tradicionales que exhiben distintos grados de subordinación femenina. Lo contrario se encuentra en los sectores populares, en los que persiste mayor rigidez en relaciones autoritarias por parte del jefe varón, que mantiene una relación de inequidad y subordinación hacia el resto de los miembros, particularmente la mujer cónyuge (García y Oliveira, 2006, p. 838).

En la infancia, los intereses profesionales giran alrededor del contexto familiar, de los patrones de socialización asociados con las diferencias de género, las amistades, las tendencias sociales, la escuela, el éxito y la satisfacción, así como de la orientación de dichos intereses (MacíasGonzález et al., 2019). A través de la intervención de la madre o previas generaciones familiares existe una transmisión generacional de conductas, que son a la vez individuales y colectivas (Blanco, 2001). La dinámica familiar recae en una variedad de fenómenos individuales, y dentro del colectivo familiar tanto en el curso de una generación como a lo largo de dos o tres generaciones, "por lo que toca al tema del amplio mundo del trabajo, entre los fenómenos que destacan se encuentran la movilidad social y ocupacional, la elección de inserciones en el mercado de trabajo y, por supuesto, las trayectorias laborales" (p. 95).

Bertaux y Thompson (1993) describen la gran importancia que tienen las mujeres en los procesos de transmisión intergeneracional, incluso en la esfera del trabajo, típicamente 
considerada como masculina. De esta manera, a pesar de que las mujeres puedan no haber realizado un trabajo extra doméstico, sobre todo en las generaciones anteriores, es fundamental su papel en la transmisión de tradiciones familiares, así como en el hecho de poder imbuir ciertas motivaciones o metas a las hijas, por ejemplo: estudiar, trabajar, a veces en contra de la tradición familiar, y es aquí en donde la mediación familiar es fundamental en la elección de vida y carrera de las mujeres.

Wilkens (1987) reportó en un análisis de empresarias exitosas que la mayoría de las mujeres investigadas tenían patrones familiares de madres fuertes, con un rol positivo y modelos que mostraban determinación y fuerza cuando venían tiempos difíciles. "La familia es sin duda una mediación de singular importancia en la modalidad y el nivel que puede asumir el trabajo femenino" (Ariza y De Oliveira, 2002, p. 49). Este cúmulo de insumos que se reciben a través de los años moldean el futuro de vida y carrera.

En esta travesía femenina la cuestión de la maternidad es decisiva para el desarrollo de una carrera laboral. "La dificultad reside en que las mujeres suelen tener los hijos en momentos cruciales de su carrera profesional, y para muchas es muy difícil después regresar al camino, incluso para las más capaces" (Hewlett, 2006, p. 319). Diversos estudios (Bringas et al., 2004; Solé y Parella, 2004) señalan que cada vez más mujeres posponen la maternidad por razones laborales.

El dilema entre el trabajo y la familia tiene diferentes implicaciones en las mujeres y no todas lo resuelven igual: existen ejecutivas que deciden dar prioridad a sus carreras sin renunciar a la vida familiar, delegando gran parte de sus responsabilidades domésticas y de cuidado en sus parejas, familias o personal de servicio (Hewlett, 2006). La familia no solo condiciona el nivel de participación de las mujeres en el mercado de trabajo, también influye en la magnitud de esta participación, el trabajo a tiempo parcial de las mujeres es una forma de conciliar familia y trabajo muy normalizada en algunos países (Moine, 2013).

Para las profesionistas con al menos un hijo, el primero y más contundente conflicto que señalan, es el que tiene que ver con las responsabilidades familiares. La estadística (Mckinsey y Company, 2007) propone que 27 por ciento de los hombres estaría dispuesto a dedicarse a las labores del hogar, sin embargo, todavía estas tareas recaen de forma desigual sobre las mujeres, en especial las relacionadas con el cuidado de dependientes (OIT, 2019).

$\mathrm{El}$ incremento de mujeres en distintas organizaciones con niveles de toma de decisiones es el resultado de los cambios generados en la configuración de las familias y en las transformaciones sociales y culturales, entre ellos se destacan el sólido aumento de la participación económica y laboral de las 
mujeres (Chávez y Ríos, 2014). Es necesario tener en cuenta que no existe un único modelo de mujer y que aún las ejecutivas comprenden diferentes ambiciones y motivaciones. Schwartz (1989), al investigar el desarrollo de carrera de ejecutivas, incluye que existen dos tipos bien diferenciados: por un lado, las que dan prioridad a sus carreras y, por otro, las mujeres orientadas a carrera y familia, y en función de estas y otras elecciones, las ejecutivas deberían ser tratadas y consideradas en forma diferente por las corporaciones.

\section{Mediación organizacional}

La presente investigación centró su interés en las mujeres ejecutivas o de alta gerencia, quienes son una cantidad pequeña de personas en la organización y son responsables de establecer políticas de operaciones, dirigen la interacción y organización de su equipo; pueden ser gerentes, subdirectoras o directoras (Villasmil, 2007). Las mediaciones de socialización y cultura organizacional que se consideran en el presente apartado se relacionan con la vida organizacional que interviene a estas mujeres.

La socialización y la cultura organizacional son una mediación fundamental en el desarrollo de vida y carrera. Esta socialización empieza desde la infancia con los discursos de los padres y el consumo de los medios de comunicación y se replantea en la edad adulta, en donde se ejercen labores mediadas por la cultura de la organización a la que pertenecen, determinadas en un contexto geográfico, cultural y económico. Shein (1988) define la socialización organizacional como la forma de ponerse al tanto en el proceso de adiestramiento en una parte de la organización o en la compañía entera; en la socialización organizacional es donde se hace vida la cultura organizacional y se encuentra entretejida la mediación de la institución. Por su parte, la cultura organizacional es el conjunto de suposiciones, valores y creencias por las cuales se rige una organización en la toma de oportunidades y decisiones (McShane y Von Glinow, 2008).

Las mujeres han entrado con fuerza al ámbito laboral y en el sustento familiar participan junto con los hombres para financiar los costos del hogar; sin embargo, este replanteamiento de la aportación económica no ha estado acompañado del mismo escenario en la cuestión doméstica, y el trabajo del hogar sigue condicionado a las mujeres, lo que las pone en una situación de desventaja (Lipovetsky, 2002; Ariza y De Oliveira, 2006; White et al., 1997).

La vida organizacional se ha tenido que adaptar con la integración de las mujeres a su plantilla y empatar sus características laborales, asumiendo que las mujeres forman parte de la institución, no solo en puestos operativos, sino como ejecutivas con responsabilidad ante grupos y en toma de decisiones. Mena (2019) describe cinco 
elementos importantes asociados a la cultura organizacional:

1. Comportamientos: las conductas y los comportamientos de las personas en sus relaciones ordinarias. Son los procesos de relación que dan vida a las organizaciones y se complementa con las maneras en que circulan las informaciones, el lenguaje que se utiliza, los rituales, las manifestaciones y las expresiones, el respeto, etcétera.

2. Normas: son las pautas y reglas que, desde el punto de vista formal e informal, en la empresa tienden a ordenar los distintos procedimientos y formas de conducta. 3. Filosofía: desde la perspectiva organizacional, se refiere a las formas en que se crean, orientan y desarrollan las estrategias y políticas de la empresa en un proceso que parte de las características de los distintos tipos de públicos (filosofía de la empresa).

4. Clima: es el ambiente interno de la organización, se genera a partir de las formas en que los empleados se relacionen entre sí y en cómo se relacionan con los públicos externos. La reputación corporativa de una organización depende en gran medida de su clima interno.
5. Valores: forman parte intrínseca de las organizaciones: "Los valores organizacionales esencialmente son cualidades de la cultura de las empresas, que son jerarquizados o asumidos de preferencia porque son percibidos (en mayor o menor grado de conciencia), como elementos indispensables para alcanzar logros colectivos" (Fundora, 2007, p. 28).

La cultura y socialización organizacional se ven entretejidos en su cotidianeidad y van adaptando sus formatos de comportamiento de acuerdo con los tiempos históricos y contextuales en donde tengan que ejercer su productividad. En la medida en que se refuerzan las conductas, se construye el discurso de la cultura de la empresa y se modelan las conductas y expectativas de los empleados (Robbins, 2004).

La vida en la organización ha contribuido de manera significativa en la construcción de lo que se considera femenino y masculino. Las emociones y sentimientos, vistos como femeninos, se consideraron ajenos a la organización por muchos años $y$, por el contrario, la racionalización fue privilegiada y socialmente planteada como neutra, aunque con una connotación masculina al interior de la organización. En la medida en que estos discursos se fueron legitimando, la presencia de la mujer fue quedando fuera de cuadro en el 
crecimiento vertical en la vida laboral (Zabludovsky, 2015).

Adhler (2002) menciona diferentes facetas en la incorporación de la mujer a la empresa. La primera, buscó que las mujeres actuaran como hombres; la segunda, que se trataran como gerentes con las mismas cualidades, sin distinción por sexo; la tercera buscó valorar a las mujeres por su sexo en sí, ya que se dieron cuenta de la importancia de las mujeres en la toma de decisiones de compra, de su fortaleza en las campañas de venta y se inclinaron a emplear mujeres; la cuarta, de mayor madurez, persigue la incorporación de las cualidades tanto de hombres como mujeres, en favor de la diversidad, la innovación y la capacidad creativa de ambas perspectivas.

El proceso en cada institución es distinto y depende de la cultura política, social, económica y empresarial de cada país, así como de cada subcultura. No es posible homologar este proceso de integración laboral femenina puesto que existen empresas en los todos los países del mundo que entremezclan todas las facetas mencionadas por Adhler (2002), sobre todo cuando son empresas locales, que tienen políticas específicas y no se adscriben a las regulaciones laborales a nivel global.

"Cada vez hay mayor conciencia de que la meritocracia (dejar que el talento llegue a la cima, independientemente de su género o procedencia) es esencial para el éxito empresarial" (Charlo y Núñez, 2012, p.89). En esta realidad laboral, las mujeres están en la competencia y "dada la rapidez con que se suscitan los acontecimientos y cambian los escenarios, la única fortaleza competitiva de los negocios ha llegado a ser el conocimiento" (Puga y Martínez, 2008, p.90), sin embargo, sigue existiendo una segregación de las mujeres en el mercado del trabajo y no hay una correlación directa en la obtención de empleos.

Este escenario desigual es un resultado cultural y se explica desde varios acercamientos. Nicolson (1997) prescribe que hay tres tipos de procesos de discriminación en las organizaciones, lo que se transforma en barreras para la vida productiva de las mujeres: 1.-Las barreras estructurales visibles, falta de apoyo para el cuidado de los niños, carencia de modelos de referencia femeninos y asesorías, actitudes sexistas y acoso sexual.

2.-Las barreras invisibles, prejuicios, creencias y conductas definidas por los hombres como exclusivas para ellos mismos. 3.-El impacto psicológico inconsciente de las organizaciones sobre las mujeres, su autoestima y la relación que viven entre el contexto y el conocimiento en las organizaciones.

Por su parte Ramos, Barberá y Sarrio (2003), indican barreras de tipo interno y externo que dificultan el ascenso de las mujeres a puestos de poder: a) barreras internas asociadas a la identidad de género femenino, b) barreras 
externas asociadas a la cultura organizacional y los estereotipos de género, c) barreras producidas por el rol reproductivo y las responsabilidades familiares.

La cultura organizacional tradicional ejerce un desarrollo diferencial según el sexo, y en términos generales, se mantiene la idea de que los cargos gerenciales no son propiamente femeninos (Hola y Todaro, 1992). La baja participación de mujeres en puestos directivos se justifica desde los factores de la cultura organizacional, la distribución y jerarquización por género; las distintas políticas de evaluación y compensación para mujeres y hombres, la poca flexibilidad en la mayoría de las organizaciones y las expectativas respecto al compromiso con la organización que le exigen confinar a un segundo término o renunciar a otro tipo de actividades, como las personales o familiares (Zabludovsky, 2015).

Las empresas más tradicionales, para no promover a las mujeres a cargos de dirección estipulan que los directivos ocupan más compromiso con la organización y las mujeres deben cumplir con el horario de salida para hacerse cargo de las responsabilidades familiares. Por su parte, las empresas más modernas enuncian el problema como una elección de la mujer, afirman que las mujeres pueden ocupar cualquier cargo, siempre que se queden hasta la hora que sea necesaria para sacar los objetivos. Con palabras distintas y en el fondo el mismo discurso, se enuncia que "las mujeres no son gerentes porque no trabajan hasta tarde, aunque puedan serlo si así lo desean y se adapten a esas reglas" (Hola y Todaro, 1992, pp. 22-23).

Gherardi (1995) explica que tradicionalmente las mujeres están ubicadas en puestos de segundo nivel o posiciones femeninas identificadas con relaciones personales, servicios de reproducción o afines y "cuando las mujeres ocupan una posición femenina, deben esforzarse por ser discretas, casi invisibles" (p. 15). Beauvoir (1981) coincide y menciona que la mujer se sabe juzgada por su indumentaria y su cuidado, por tener una buena apariencia en el trabajo, entiende que se espera de ella una conducta dócil y una representación femenina en todo momento. Gherardi (1995) utiliza el término francés bon ton, para enunciar lo que es adecuado en la etiqueta de socialización organizacional.

El triple papel de las mujeres: esposa, madre y directiva, representa un problema crucial para asumir puestos que demandan disponibilidad casi total. Las mujeres se enfrentan a diversas problemáticas al querer entrar al mundo corporativo: existe el techo de cristal (Davidson y Cooper, 1992), que les impide acceder a puestos mejores, por los prejuicios y estereotipos de género; el techo de cemento (Bejarano, 2007; Equal, 2012) que es el que ellas mismas se ponen al no querer arriesgar su vida personal, por las responsabilidades que implica un mejor puesto 
en la organización; así también el terreno pegajoso o suelo pegajoso, que son los puestos de baja responsabilidad al que las mujeres acceden en sus inicios, tienen un bajo salario y en el cual se mantienen por largo tiempo, a favor del equilibrio de su tiempo, trabajo y familia (Camarena y Saavedra, 2016; Equal, 2012).

En la segregación horizontal y en la vertical se encuentran otros bloqueos; la primera se refiere a la posición del trabajo de las mujeres en determinados sectores (como servicios personales), en posiciones a nivel de subordinación, y la segunda, a la poca representación de las mujeres en los puestos de alta responsabilidad, los cuales tienen un mayor reconocimiento profesional (Camarena y Saavedra, 2016; Equal, 2012).

Las mujeres han logrado romper barreras (Nicolson, 1997; Ramos, Barberá y Sarrio 2003), techos (Chinchilla y León, 2004; Equal, 2012) y otras creencias, a través de su trayectoria en la organización. Metz (2005) define que las mujeres profesionistas ejecutivas invierten más cantidad de horas en el trabajo, tienen amplia experiencia laboral, ambición, buen nivel de educación, entrenamiento constante y aprovechan las oportunidades que se presentan en su carrera. Estas mujeres rompen la frontera de la norma en los tiempos y espacios y generan su propio lugar, en un tercer espacio.

Zabludovsky (2009) encuentra que los funcionarios y directivos trabajan entre 45 y 50 horas a la semana, esto significa que se tiene que priorizar el trabajo, ante otras áreas personales. La mayoría de los directivos, son vistos como adictos al trabajo, ya que tienen que dedicar una gran cantidad de horas para ejecutar los puestos que ejercen. Por estas circunstancias, en el momento que las mujeres desean ingresar a las carreras ejecutivas en el sector privado, se encuentran que, a nivel simbólico, la empresa considera que cuando contrata a un hombre casado está empleando a dos personas (a él mismo y a su esposa) y que cuando emplea a una mujer casada, por las responsabilidades que esta tiene en el hogar, se está ocupando a tan solo media persona (Zabludovsky, 2007).

Las madres de niños pequeños se enfrentan a una penalización en los cargos de liderazgo vinculada con la maternidad, registran las tasas más bajas de participación en los cargos directivos y de liderazgo (solo $25.1 \%$ del personal directivo con niños menores de 6 años de edad son mujeres) en comparación con sus homólogos masculinos (74.9\% del personal directivo con niños menores de 6 años son hombres) y con los hombres y mujeres sin niños pequeños $(31.4 \%$ de los cargos directivos son mujeres, y el $68.6 \%$ son hombres) (OIT, 2019).

\section{Mediación organizacional en México}

En México, sólo 10 por ciento de los puestos directivos están ocupados por mujeres, lo que 
representa el lugar 81 de 144 países en cuanto a la inclusión de mujeres en puestos ejecutivos (Ochoa, 2018). Muchas mujeres mexicanas se enfrentan a grandes bloqueos que les impiden participar plenamente en el mercado laboral. Estos incluyen: la carga del trabajo no remunerado (las mexicanas dedican cuatro horas diarias más al trabajo no remunerado que los hombres); los tradicionales roles de género; y la escasez de políticas de conciliación entre trabajo y vida familiar, específicamente la insuficiente oferta de servicios de cuidado infantil y de prácticas laborales flexibles (OCDE, 2011).

Bejarano (2007) describe el costo por equilibrar la vida doméstica y laboral, sobre todo en los primeros meses y años de los hijos, así también narra que las mujeres que logran acceder a un cargo directivo sufren diversos costos físicos, emocionales o prejuicios sociales, ya que para conseguirlo debieron dejar de lado mayores responsabilidades familiares, al mismo tiempo que rompieron barreras personales (roles familiares, autoestima femenina), organizacionales (grupos de poder, insolidaridad femenina) y sociales (cultura machista mexicana y cambio social), para poder acceder a puestos de dirección en la organización (Gaete et al., 2019).

Si se busca a las mujeres en los principales cargos ejecutivos de las grandes empresas en México, se encontrarán en direcciones de recursos humanos y compras, en segundo y tercer lugar, en las direcciones de comunicación, relaciones públicas y en actividades relacionadas con ventas y finanzas (Zabludovsky, 1997, 2012). Gherardi (1995) las categoriza en puestos de segundo nivel, de relaciones personales, recursos humanos $\mathrm{y}$ servicios de reproducción. Por otro lado, los hombres están en la categoría de organizaciones de verdad en puestos de ingeniería, informática, comercio y finanzas (Gherardi, 1995). El área de finanzas se traslapa para ambos géneros.

La participación de las mujeres en los cargos de funcionarios y gerentes en México se ha incrementado del 10 por ciento en 1991 al 24.8 por ciento, en 2006 (Chávez y Ríos, 2014), al 37 por ciento, en el 2019 (Campio, 2020). A pesar de esto, se han encontrado evidencias de una clara preferencia por parte de los empleadores por contratar más hombres que mujeres en los cargos jerárquicos, e incluso la preferencia por mujeres solteras y sin hijos (Chávez y Ríos, 2014). Camarena y Saavedra (2018) plantean, que menos del 5 por ciento de los cargos directivos de empresas que cotizan en la Bolsa Mexicana de Valores son ocupados por mujeres. Según Martínez (2004) la poca presencia de mujeres en la dirección de las organizaciones empresariales se despliega de dos cuestiones básicas:

1) La presencia de empresas dirigidas por mujeres no tiene el peso económico suficiente para que la élite directiva las considere como 
candidatas potenciales a un puesto de dirección.

2) Las mujeres empresarias no participan activamente, ni muestran interés por hacer política al interior de las cámaras patronales.

Las mujeres consideran que los mayores obstáculos que detienen su carrera profesional, por los cuales hay poca presencia femenina en la alta dirección, son motivos personales (De Anca y Aragón, 2007), la dificultad para entrelazar el trabajo directivo con las responsabilidades familiares significa un obstáculo importante en el ascenso y promoción de las mujeres.

\section{Reflexiones finales}

En síntesis, es importante que cualquier acción para incentivar la presencia de mujeres en puestos directivos requiere como condición obligatoria, disminuir las responsabilidades familiares, y compartir con los varones las responsabilidades domésticas (Barberà et al., 2003; Kauffman, 2009). Algunas investigaciones han comprobado que los casos en los que los hombres comparten de manera más equitativa el trabajo de cuidados no remunerado con las mujeres, estas tienen una mayor participación en cargos directivos (Charlo y Núñez, 2012; OIT, 2019).
En México, así como en todo el mundo, la cultura organizacional es matizada por la cultura social e histórica de cada país. El discurso público maneja la equidad laboral y doméstica, sin embargo, al interior de los hogares y en los puestos de alta dirección, aún se viven grandes rezagos ideológicos y los estereotipos tradicionales coexisten en la sociedad mexicana. Romper estos estereotipos del hombre como proveedor y la mujer como encargada de la casa (Camarena y Saavedra, 2016) es un reto constante y plantea desafíos a nivel individual y colectivo para las mujeres y los hombres. García (2014) plantea el cambio de encuadre como propuesta de cambio en los medios masivos de comunicación, en donde se representen mujeres con capacidades de liderazgo y características androcéntricas que coincidan con los perfiles de alta gerencia.

En relación a los medios masivos de comunicación estos definen a la mujer como joven, bella, femenina (Wagner y Wodak, 2006), subordinada, sumisa y dependiente (Rocha-Sánchez y Díaz-Loving, 2005; Bonavitta y Garay Hernández, 2014; García, 2014) características que no compaginan con la realidad de las mujeres ejecutivas, quienes están en una edad madura y tienen características androcéntricas o referenciadas con el género masculino (Koller, 2004; García, 2014). Los medios masivos de comunicación son indisociables de la vida cotidiana y forman parte de las mediaciones de la sociedad promoviendo los estereotipos de género, sin 
embargo, no se manifiestan como los principales referentes de éxito en mediación de las mujeres de alta gerencia, sino como formadores de estereotipos contrarios ante su capacidad de liderazgo (García, 2014).

Los resultados de la OIT (2019) soportan este escenario y definen que los avances para disminuir las brechas de género se han detenido y en algunos casos, se ha observado un retroceso, la organización describe que en los últimos 20 años el avance no ha sido significativo, lo cual demuestra la incongruencia entre el discurso y la vida práctica de las mujeres mexicanas (Zabludovsky, 2015; Gaete et al., 2019; OIT, 2019).

\section{Referencias bibliográficas}

Adhler, N. (2002). Global managers: No longer men alone. International Journal of Human Resource Management, 13(5), pp. 743-760.

Ariza, M., y De Oliveira, O. (2006). Regímenes sociodemográficos y estructura familiar: los escenarios cambiantes de los hogares mexicanos. Estudios Sociológicos, 24(70), pp. 3-30. http://www.jstor.org/stable/40421023.

Anderson, C. (2006). The long tail: Why the future of business is selling less of more. Hyperion.

Ariza, M., y De Oliveira, O. (2002). Cambios y continuidades en el trabajo, la familia y la condición de las mujeres. Estudios sobre las mujeres y las relaciones de género en México: aportes desde diversas disciplinas, 43-86.

Astin, H. S. (1984). The meaning of work in women's lives: A sociopsychological model of career choice and work behavior. The Counseling Psychologist, 2, pp. 117-126.

Barberà, E., Ramos, A., y Sarrió, MT. (2003). Mujeres directivas, espacio de poder y relaciones de género. Anuario de psicología/The UB Journal of psychology, 34(2), pp. 267-278

Bejarano, Z. M. (2007). Entre lo público, privado y doméstico: mujeres bajo un techo de cristal. Revista GénEros, $\quad$ 13(36), $\quad$ pp. 60-68. http://bvirtual.ucol.mx/descargables/319 entre lo_publico.pdf.

Bernal, M. C., y Taracena, E. (2013). Talento femenino en la alta dirección en México. LID.

Bertaux-Wiame, I. (1993). The pull of family ties: Intergenerational relationships and life paths. En D. Bertaux y P. Thompson (Eds.), Between generations: Family models, myths and memories. Oxford University Press.

Blanco, M. (2001). Trayectorias laborales y cambio generacional: mujeres de sectores medios en la ciudad de México. Revista Mexicana de Sociología, 63(2), pp. 91-111.

Global Media Journal México 17(33). julio - diciembre 2020. Pp. 50-71. 
Bradford, L., Buck, J. L., y Meyers, R. A. (2001). Cultural and parental communicative influences on the career success of white and black women. Women's Studies in Communication, 23, pp. 194-217.

Bringas, Á, Espinosa, S., Islas, S., Ezcurdia, C., y Torres, E. (2004). Nuevas maternidades o la desconstrucción de la maternidad en México. Debate Feminista, 30, pp. 55-86. https://www.jstor.org/stable/42624831.

Bonavitta, P., y Garay Hernández, J. de. (2014). De estereotipos, violencia y sexismo: la construcción de las mujeres en los medios mexicanos y argentinos. Anagramas. Rumbos y Sentidos de la Comunicación, 9(18), pp. 15-29.

Camarena, M., y Saavedra M. (2016). Un estudio de las PYME lideradas por mujeres en Latinoamérica. Revista Universitaria Ruta, 18(1), pp. 1-27. https://revistas.userena.cl/index.php/ruta/article/view/789.

Camarena, M., y Saavedra, M. (2018). El techo de cristal en México. Revista La Ventana, 5(47), pp. 312-347. http://revistalaventana. cucsh.udg.mx/index.php/LV/article/view/6680.

Campio, H. (2020). México alcanza el máximo histórico de mujeres en puestos de alta dirección. $\begin{array}{lllll}\text { Grand } & \text { Thornton, } & 8 & \text { de } & \text { diciembre }\end{array}$ https://www.grantthornton.mx/prensa/marzo_20202/Mexico_alcanza_el_maximo_historico _de_mujeres_en_puestos_de_alta_direccion/.

Cárdenas de Santamaría, M. C., Eagly, A. H., Heller, L., Salgado, E., Jaúregui, K., y Goode, W. (2013). Claves para el ascenso de las altas ejecutivas en América Latina. INCAE Business Review, 2(9), pp. 52-56. http://www.latam-studies.com/INCAE2.html.

Charlo Molina, M. J., y Núñez Torrado, M. (2012). La mujer directiva en la gran empresa española: perfil, competencias y estilos de dirección. Estudios Gerenciales, 28(124), pp. 87-105.

Chávez, N. M., y Ríos, H. (2014). Discriminación salarial por género “efecto techo de cristal”. Caso: siete áreas metropolitanas de Colombia. Dimensión Empresarial, 12(2), pp. 29-45.

Chinchilla, N., y León, C. (2004). La ambición femenina. Como reconciliar trabajo y familia. Aguilar.

Coller, X. (1991). Roles Familiares y Mercados De Trabajo. Revista de Sociología, (36), pp. 93-114. http://dx.doi.org/10.5565/rev/papers/v36n0.1589.

Cuadrado G. (2007). Estereotipos de género. En J. F. Morales, E. Gaviria, M. Moya, y I. Cuadrado (Coords.), Psicología social (3 ${ }^{\mathrm{a}}$ ed., pp. 243-266). McGraw-Hill.

Davidson, M. J., y Cooper, C. L. (1992). Shattering the glass ceiling: The woman manager. Paul Chapman Publishing.

Global Media Journal México 17(33). julio - diciembre 2020. Pp. 50-71. 
De Anca, C., y Aragón, S. (2007). La mujer directiva en España: catalizadores e inhibidores en las decisiones de trayectoria profesional. Academia. Revista Latinoamericana de Administración, (38), 45-63

De Beauvoir, S. (1981). El segundo sexo. Siglo XX.

De los Ríos, P, M. J., y Martínez, R. J. (1997). La mujer en los medios de comunicación. Comunicar: Revista Científica de Comunicación y Educación, 5(9), pp. 97-104.

Del Real, L. P., y Maeda, K. (2020). Autorrepresentación de las mujeres en la cultura organizacional. ResearchGate.

Preprint. https://www.researchgate.net/publication/346682298_Autorrepresentacion_de_las_mujeres _en_la_cultura_organizacional.

Demarchi, G., Aguirre, M., Yela, N., y Viveros, E. (2015). Sobre la dinámica familiar. Revisión documental. Repositorio Universidad de La Costa, 6(2), pp. 117-138. https://repositorio.cuc.edu.co/bitstream/handle/11323/3107/SobRe\%201a\%20din\%c3\%almi ca\%20familiaR.\%20ReviSi\%c3\%b3n\%20documental.pdf?sequence=1\&isAllowed=y.

Equal. (2012). Guía para la incorporación de políticas de igualdad de género en las PYMES. Delta Emprén. www.surt.org/accions/guia_delta_igualdad_pimes.pdf.

Fundora, O. (2007). Diagnóstico de valores personales y organizacionales en la Empresa Comercial SUME [Tesis de maestría]. Facultad de Comunicación de la Universidad de La Habana.

Gaete, R., Álvarez, J., y Ramírez, M. (2019). Reflexiones y experiencias de profesoras-investigadoras mexicanas sobre el techo de cristal. Calidad en la educación, (50), pp. 457-491. https://dx.doi.org/10.31619/caledu.n50.494.

García, B., y Oliveira, O. (2006). Una obra sobre las dinámicas familiares en México. Economía, sociedad y territorio, 6(23), pp. 833-848.

García, V. (2014). Influencia de la televisión en la creación de estereotipos de género y en la percepción social del liderazgo femenino: la importancia de la táctica de reencuadre para el cambio social. Ciencia Politica, 9(18), pp. 47-66.

Gherardi, S. (1995). Gender, symbolism and organizational cultures. TPB.

Herrera, S. L., y Romero, M. N. (2014). Female experiences and imaginary that naturalize the domestic violence. Index de Enfermería, 23(1-2), pp. 26-30. https://dx.doi.org/10.4321/S1132-12962014000100006.

Hewlett, S. (2006). Off ramps and on ramps: Keeping talented women on the road to success. Harvard Business School Press.

Hola, E., y Todaro, R. (1992). Los mecanismos de poder: hombres y mujeres en la empresa moderna. Grupo Editor Latinoamericano.

Global Media Journal México 17(33). julio - diciembre 2020. Pp. 50-71. 
Inegi (Instituto Nacional de Estadística y Geografía). (2020). Estadísticas a propósito del Día Internacional de la Mujer. Comunicado de Prensa núm. 127/20, pp. 1-2. https://www.inegi.org.mx/contenidos/saladeprensa/aproposito/2020/mujer2020_Nal.pdf.

Kauffman, A. (2010). Identidades en transformación: mujeres y hombres directivos en España. En Ponencias presentadas durante el XXII Seminario Iberoamericano de Sociología de las Organizaciones, Salto, 2009, pp. 520-572.

Koller, V. (2004). Metaphors in Business Magazines. Palgrave Macmillan.

Lair, D. J. (2007). Rethinking the "organizational" in organizational socialization research: From ontological agent to discursive domain (s). National Communication Association annual meeting.

Langellier, K. M., y Peterson, E. E. (2006). “Somebody's got to pick eggs”: Family storytelling about work. Communication Monographs, 73, pp. 468-473.

Lipovetsky, G. (2002). La tercera mujer. Anagrama.

Lucas, K. (2011). Blue-collar discourses of workplace dignity: Using outgroup comparisons to construct positive identities. Management Communication Quarterly, 25(2), pp. 353-374. https://doi.org/10.1177/0893318910386445.

Machín-Rincón, L., Cifre, E.; Domínguez-Castillo, P., y Segovia-Pérez, M. (2020). I am a leader, I am a mother, I can do this! The moderated mediation of psychological capital, work-family conflict, and having children on well-being of women leaders. Sustainability, 12(5), 1-22. https://doi.org/10.3390/su12052100.

Martín-Barbero, J. (1986). La cultura como mediación: comunicación, política y educación. Matrices culturales, pp. 201-211.

Nicolson, P. (1997). Poder, género y organizaciones. ¿Se valora a la mujer en la empresa? Narcea.

Macías-González, G., Caldera-Montes. J., y Salán-Ballesteros, M. (2019). Orientación vocacional en la infancia y aspiraciones de carrera por género. Convergencia, 26(80). https://doi.org/10.29101/crcs.v26i80.10516.

Mckinsey y Company (2007). Women matter, gender diversity, a corporate performance driver. https://www.mckinsey.com/business-functions/organization/our-insights/gender-diversitya-corporate-performance-driver.

McShane, S., y Glinow V. (2008). Organizational Behavior. McGraw Hill/Irwin.

Mena Méndez, D. (2019). La cultura organizacional, elementos generales, mediaciones e impacto en el desarrollo integral de las instituciones. Pensamiento y gestión, 46, pp. 11-47.

Global Media Journal México 17(33). julio - diciembre 2020. Pp. 50-71. 
Metz, I. (2005). Advancing the careers of women with children. Career Development International, $10(3)$ pp.

228-245. https://www.emerald.com/insight/content/doi/10.1108/13620430510598346/full/html.

Moine, M. B. (2013). Investigación científica: factores que inciden en el acceso de las mujeres a cargos de responsabilidad en la estructura organizacional. Ciencias económicas, 10(1), pp. 21-45.

Ochoa, C. (2018). En altos puestos, solo 10\% de las mujeres en México. Milenio 2020. https://www.milenio.com/negocios/en-altos-puestos-solo-10-de-las-mujeres-en-mexico.

Ochs, E. (1988). Culture and language development: Language acquisition and language socialization in a Samoan village. Cambridge University Press.

OIT (Organización Internacional del Trabajo). Servicio de género, igualdad y diversidad (GED) y ILOAIDS. (2019). Un paso decisivo hacia la igualdad de género. https://www.ilo.org/gender/lang--en/index.htm.

OCDE (Organización para la Cooperación y el Desarrollo Económico). (2011). Estudios económicos de la OCDE: México 2011. OECD Publishing. http://dx.doi.org/10.1787/9789264115934-es.

Orozco, G. (1991, primavera). Recepción televisiva: tres aproximaciones y una razón para su estudio. Cuadernos de Comunicación y Prácticas Sociales. Estudios sobre las Culturas Contemporáneas, IV(14), pp. 319-322.

Orozco, G. (1997). La investigación en comunicación desde la perspectiva cualitativa. Instituto Mexicano para el Desarrollo Comunitario.

O’Neil, D. A., y Bilimoria, D. (2005). Women's career development phases: Idealism, endurance, and reinvention. Career Development International, 10(3), pp. 168-189.

Padilla, M. R. (2005). Ser mujer se aprende, enseña, disfruta y sufre: Telenovela, cultura e identidad de género. Culturales, 1(1), pp. 143-176.

Parsons, T. (1955). The American family: Its relations to personality and to the social structure. En Family, socialization and interaction process, 3-33. Routledge.

Paugh, A. L. (2005). Learning about work at dinnertime: Language socialization in dual-earner American families. Discourse and Society, 16, pp. 55-78.

Puga, J., y Martinez, L. (2008). Competencias directivas en escenarios globales. Estudios Gerenciales, 24(109), pp. 87-103. http://www.icesi.edu.co/revistas/index.php/estudios_gerenciales/article/view/277/275.

Robbins, S. (2004). Comportamiento organizacional. Prentice Pearson Hall.

Rocha-Sánchez, T. E., y Díaz-Loving, R. (2005). Cultura de género: la brecha ideológica entre hombres y mujeres. Anales de Psicología/Annals of Psychology, 21(1), pp. 42-49.

Global Media Journal México 17(33). julio - diciembre 2020. Pp. 50-71. 
Serravallo, V. (2004). Less alienated labor: A source of hope-filled mobility socialization? Journal of Contemporary Ethnography, 33, pp. 368-405.

Schwartz, F. N. (1989). Management women and the new facts of life. Harvard Business Review, 67(1), pp. 65-76.

Shein, E. (1998). La cultura empresarial y el liderazgo. Una Visión Dinámica. Plaza y Janés Editores, S.A.

Simpson, R. L. (1962). Parental influence, anticipatory socialization, and social mobility. American Sociological Review, 27, pp. 517-522.

Solé, C., y Parella, S. (2004). Nuevas expresiones de la maternidad. Las madres con carreras profesionales exitosas. Revista Española de Sociología, (4), pp. 67-92.

Vega, A. (2006). Género y recepción televisiva: la interacción de las sujetas y los sujetos con y mediada por la televisión. Revista mexicana de ciencias politicas y sociales, 48(197), pp. 6781.

Velázquez, L. (2012). Carrera y desarrollo profesional. Grupo Fractal Editores.

Villasmil, Jonathan (2007). Gerencia y Liderazgo [documento].

Wagner, I., y Wodak, R. (2006). Performing success: Identifying strategies of self-presentation in women's biographical narratives. Discourse \& Society, 17(3), pp. 385-411.

White., B., Cox, C., Cooper, C. L. (1997). A portrait of successful women. Women in Management Review, 12(1), pp. 27-34. https://doi.org/10.1108/09649429710162910.

Wilkens, J. (1987). Her own business: Success secrets of entrepreneurial women. McGraw-Hill.

World Economic Forum. (2014). The global gender gap report 2014. http://www3.weforum.org/docs/WEF_GGGR_2014.pdf.

World Economic Forum. (2018). The global gender gap report 2018. http://www3.weforum.org/docs/WEF GGGR 2018.pdf.

Zabludovsky, G. (1997, enero-abril). Presencia de las mujeres ejecutivas en México. Sociológica, 12(33), pp. 73-99.

Zabludovsky, G. (2007, primer semestre). México: mujeres en cargos de dirección del sector privado. Academia. Revista Latinoamericana de Administración, (38), pp. 9-26.

Zabludovsky, G. (2015). Las mujeres en los ámbitos de poder económico y político de México. Revista Mexicana de Ciencias Políticas y Sociales, 60(223), pp. 61-94.

Global Media Journal México 17(33). julio - diciembre 2020. Pp. 50-71. 